\title{
Study on the Impact of "Health Qigong•WuQinxi" on the Physical Health of the University Students
}

\author{
Xiang Bin \\ Physical Education Department, Xi'an University of Science and Technology, Xi'an, Shaanxi 710054 \\ xiangbin@guigu.org
}

\begin{abstract}
This study based on documents and materials, interview, experiment and data statistical analysis, as well as by the means of "Health Qigong•WuQinxi", and carries on a 4-month exercise intervention aiming at the university students, furthermore to have a comparison of their level scores on physical shape, physiological indicator, before and after the experiment. And we come to a conclusion through this 4-month exercise, when concerns to physical shape and physiological indicator: we can see a significant difference on WHR, body mass index and vital capacity $(\mathrm{p} \leqslant 0.01)$; as well as waist circumference, pulse, systolic blood pressure, diastolic blood pressure, sit and reach, grip strength $(\mathrm{p} \leqslant 0.05)$. So we note that long-term "Health Qigong•WuQinxi" practice can improve the physical health of the university students, and assess the students who failed to meet health standards physically, and finally realize our hope promoting the comprehensive development of university students.
\end{abstract}

Keywords: Health Qigong $\bullet W u Q i n x i, \quad$ University Students, Physical Health

\section{INTRODUCTION}

"Health Qigong•WuQinxi" can combine the body movements, the breathing and aspiration and the psychological adjustment together harmoniously. It can not only stretch human body and ease joints, but also can have an adjustment effect on the sub-health of human body. "Health Qigong•WuQinxi" is an aerobic exercise with a moderate intensity, and thus is suitable of most people to exercise.

\section{Selection and Significance of Body Physiques and Physiological Indexes}

Height is a major physique index to reflect the growth of human skeleton and the longitudinal height of human bodies. Weight is a general index to reflect the lateral growth, circumference, width, thickness and fitness. The height and weight are combined to compose of BMI; the weight/height index $(2 \mathrm{~kg} / \mathrm{m} 2)$ is used to evaluate the body shape, growth and nutrition. $\mathrm{BMI} \leqslant 20$ (towards thin) and $\mathrm{BMI} \geqslant 26.5$ (towards fat) are usually taken as the normal range of weight/height index. In general, a standard to measure the body's fat and thin as well as health is used as a physique index to evaluate an individual development, making it better judge the physical condition of an individual as a whole ${ }^{[1]}$. Multiple documents suggest that BMI index has a close connection with many body diseases ${ }^{[2,3]}$.

The cardiovascular system is one of the most important systems of human body. Pulse, diastolic blood pressure, systolic blood pressure and step experiment are important indexes to measure the functions of the cardiovascular system. The lung capacity has a tie with age, gender, body surface area, thoracic size and respiratory muscle development, and is different individually; its absolute value can't reflect the ventilation function of human body comprehensively, and thus its relative value that is the lung capacity/weight index $(\mathrm{ml} / \mathrm{kg})$ is adopted to evaluate the lung ventilation function ${ }^{[4]}$. In the sit-and-reach test under a rest condition, the possible movement spans of the body, waist and hip joints can reflect the extensibility and elasticity of the joints, ligaments and muscles of these body parts and the development of the body flexibility quality ${ }^{[5]}$. The balanced capacity is an ability to control 
the body gravity center within a smaller supporting area, and reflects the ability of body to s coordinate the proprioceptor and vision from the vestibular organs, muscles, tendons and the joint inside and outside. The abdominal muscle endurance is tested by the sit-ups ${ }^{[6]}$.

\section{Research Objects and Methods}

The undergraduates were the research objects. 160 students at Xi'an University of Science and Technology were randomly selected as the experimental objects. Among them, the male students were 80 and the average age were between $21.8 \pm 1.7$; the female students were 80 and the average age were between $20.5 \pm 2.1$. 56 students (45 males and 11 females) were from school of energy engineering; 47 students ( 8 males and 39 females) were school of management; 32 students (22 males and 10 females) were from school of mechanical engineering; 25 students (5 males and 20 females) were from school of English language. Also, 92 students were freshmen; 68 students were sophomores. The experimental

Table 1: Comparison on the Body Physique Indexes of Referential Group and Experimental Group before the Experiment $(\mathrm{M} \pm \mathrm{SD})$

\begin{tabular}{ccccc}
\hline \multirow{2}{*}{$\begin{array}{c}\text { Physique } \\
\text { Index }\end{array}$} & \begin{tabular}{c} 
Males \\
Referential Group \\
\cline { 2 - 5 }
\end{tabular} & $\begin{array}{c}\text { Experimental Group } \\
(40)\end{array}$ & $\begin{array}{c}\text { Referential Group } \\
(40)\end{array}$ & $\begin{array}{c}\text { Experimental Group } \\
(40)\end{array}$ \\
\hline Height $(\mathrm{cm})$ & $171.53 \pm 5.06$ & $170.98 \pm 6.06$ & $158.32 \pm 4.78$ & $159.46 \pm 5.56$ \\
Weight $(\mathrm{kg})$ & $62.24 \pm 9.06$ & $60.24 \pm 9.05$ & $52.17 \pm 8.35$ & $51.25 \pm 7.32$ \\
BMI(kg/m2) & $21.45 \pm 4.27$ & $20.57 \pm 3.63$ & $20.57 \pm 3.28$ & $20.16 \pm 2.26$ \\
Waist & $73.26 \pm 6.23$ & $73.22 \pm 5.21$ & $63.59 \pm 7.19$ & $63.59 \pm 2.31$ \\
Hip & $88.27 \pm 7.26$ & $86.76 \pm 6.14$ & $89.17 \pm 5.34$ & $88.32 \pm 4.28$ \\
WHR & $0.83 \pm 0.18$ & $0.84 \pm 0.21$ & $0.71 \pm 0.26$ & $0.72 \pm 0.25$ \\
F\% & $23.59 \pm 10.17$ & $24.12 \pm 10.89$ & $35.39 \pm 10.19$ & $34.98 \pm 10.86$ \\
\hline
\end{tabular}

Note: F\% represents a sum of the upper-arm sebum thickness and scapular sebum thickness

The results of the body physique and physiological participants were sound without great diseases, but had not received a systemic exercise. The research objects were randomly divided into experimental group and referential group; 80 students (40 males and 40 females) were districted to each group; the experimental group conducted a "Health Qigong•WuQinxi" exercise for 16 weeks, and its members were tested in the body physique index and physiological index before and after the experiment. The purpose of this research was to study the impact of Health Qigong•WuQinxi" on the physical health of students. The research methods included the documentation, interviewing experts, experimentation, data statistics and logical analysis.

\section{Results and Analysis}

\subsection{Body Physiques and Physiological Indexes}

4.1.1 Comparative Analysis on Body Physique and Physiological Indexes of Referential Group and Experimental Group before the Experiment 
Table 2: Comparison on the Physiological Indexes of Referential Group and Experimental Group before the Experiment $(\mathrm{M} \pm \mathrm{SD})$

\begin{tabular}{ccccc}
\hline \multirow{2}{*}{$\begin{array}{c}\text { Physiological } \\
\text { Index }\end{array}$} & $\begin{array}{c}\text { Males } \\
\text { Referential Group }\end{array}$ & \begin{tabular}{c} 
Experimental Group \\
\cline { 2 - 5 }
\end{tabular} & $(40)$ & $\begin{array}{c}\text { Referential Group } \\
\text { Experimental Group }\end{array}$ \\
\hline Pulse (times/min) & $80.72 \pm 5.19$ & $81.12 \pm 7.44$ & $82.19 \pm 9.12$ & $83.52 \pm 8.57$ \\
PP (mmHg) & $40.59 \pm 10.26$ & $42.12 \pm 11.59$ & $38.33 \pm 10.34$ & $40.02 \pm 10.25$ \\
SBP ((mmHg) & $122.31 \pm 11.02$ & $121.12 \pm 10.29$ & $115.76 \pm 11.29$ & $114.22 \pm 9.17$ \\
DBP (mmHg) & $74.26 \pm 10.19$ & $76.89 \pm 8.12$ & $71.66 \pm 10.82$ & $72.15 \pm 7.89$ \\
VCwI & $52.19 \pm 10.62$ & $51.26 \pm 10.29$ & $40.72 \pm 10.17$ & $40.33 \pm 10.18$ \\
SE & $43.45 \pm 6.29$ & $43.27 \pm 5.21$ & $41.12 \pm 3.24$ & $40.18 \pm 4.27$ \\
SR (cm) & $8.57 \pm 7.26$ & $7.82 \pm 7.23$ & $12.52 \pm 7.19$ & $11.72 \pm 5.97$ \\
Grip (kg) & $37.74 \pm 8.33$ & $38.92 \pm 7.83$ & $21.47 \pm 6.54$ & $21.53 \pm 6.10$ \\
SSFEC (s) & $32.69 \pm 11.23$ & $31.29 \pm 10.12$ & $29.39 \pm 11.20$ & $27.17 \pm 9.27$ \\
sit-up (times /min) & $16.76 \pm 5.89$ & $16.23 \pm 4.79$ & $6.58 \pm 3.27$ & $5.17 \pm 2.35$ \\
\hline
\end{tabular}

Note: Pulse Pressure (PP), Diastolic Blood Pressure (DBP), Systolic Blood Pressure (SBP), Vital Capacity/Weight Index (VCWI), Steps Experiment (SE), sit-and-reach (SR), Standing with a Single Foot and Eyes
Closing (SSFEC)

4.1.2 Comparative Analysis on Body Physique and Physiological Indexes of Referential Group before and after the Experiment

Table 3: Comparison on the Body Physique Indexes of Referential Group before and after the Experiment $(M \pm S D)$

\begin{tabular}{ccccc}
\hline \multirow{2}{*}{$\begin{array}{c}\text { Physique } \\
\text { Index }\end{array}$} & $\begin{array}{c}\text { Mefore Experiment } \\
\text { (40) }\end{array}$ & After Experiment & Before Experiment & After Experiment \\
\cline { 2 - 5 } & $171.53 \pm 5.06$ & $171.98 \pm 6.76$ & $158.32 \pm 4.78$ & $159.16 \pm 3.58$ \\
Height $(\mathrm{cm})$ & $62.24 \pm 9.06$ & $63.19 \pm 9.35$ & $52.17 \pm 8.35$ & $53.36 \pm 7.82$ \\
Weight $(\mathrm{kg})$ & $21.45 \pm 4.27$ & $21.34 \pm 3.93$ & $20.57 \pm 3.28$ & $21.06 \pm 3.29$ \\
BMI(kg/m2) & $73.26 \pm 6.23$ & $73.22 \pm 5.21$ & $63.59 \pm 7.19$ & $65.33 \pm 2.31$ \\
Waist & $88.27 \pm 7.26$ & $86.71 \pm 6.14$ & $89.17 \pm 5.34$ & $90.42 \pm 5.38$ \\
Hip & $0.83 \pm 0.18$ & $0.84 \pm 0.23$ & $0.71 \pm 0.26$ & $0.72 \pm 0.19$ \\
WHR & $23.59 \pm 10.17$ & $24.12 \pm 10.89$ & $35.39 \pm 10.19$ & $34.98 \pm 10.86$ \\
F\% & & & &
\end{tabular}

Note: F\% represents a sum of the upper-arm sebum thickness and scapular sebum thickness
Comparisons on the body physique and physiological indexes of referential group before and 
after the experiment are shown respectively in table 3 and table 4 .

Table 4: Comparison on the Physiological Indexes of Referential Group before and after the Experiment $(M \pm S D)$

\begin{tabular}{|c|c|c|c|c|}
\hline \multirow{2}{*}{$\begin{array}{l}\text { Physiological } \\
\text { Index }\end{array}$} & \multicolumn{2}{|c|}{ Males } & \multicolumn{2}{|c|}{ Females } \\
\hline & $\begin{array}{l}\text { Before Experiment } \\
\text { (40) }\end{array}$ & $\begin{array}{c}\text { After Experiment } \\
\text { (37) }\end{array}$ & $\begin{array}{c}\text { Before Experiment } \\
\text { (40) }\end{array}$ & $\begin{array}{c}\text { After Experiment } \\
\text { (39) }\end{array}$ \\
\hline Pulse (times $\mathrm{min}$ ) & $80.72 \pm 5.19$ & $81.39 \pm 7.96$ & $82.19 \pm 9.12$ & $83.17 \pm 9.43$ \\
\hline $\mathrm{PP}(\mathrm{mmHg})$ & $40.59 \pm 10.26$ & $41.29 \pm 10.19$ & $38.33 \pm 10.34$ & $39.07 \pm 10.35$ \\
\hline $\mathrm{DBP}((\mathrm{mmHg})$ & $122.31 \pm 11.02$ & $121.22 \pm 10.36$ & $115.76 \pm 11.29$ & $114.72 \pm 9.59$ \\
\hline $\mathrm{SBP}(\mathrm{mmHg})$ & $74.26 \pm 10.19$ & $74.78 \pm 8.36$ & $71.66 \pm 10.82$ & $72.75 \pm 9.98$ \\
\hline VCWI & $52.19 \pm 10.62$ & $51.75 \pm 11.29$ & $40.72 \pm 10.17$ & $42.73 \pm 10.38$ \\
\hline SE & $43.45 \pm 6.29$ & $43.19 \pm 5.72$ & $41.12 \pm 3.24$ & $40.38 \pm 5.29$ \\
\hline $\mathrm{SR}(\mathrm{cm})$ & $8.57 \pm 7.26$ & $7.89 \pm 8.29$ & $12.52 \pm 7.19$ & $13.22 \pm 5.88$ \\
\hline Grip (kg) & $37.74 \pm 8.33$ & $38.28 \pm 8.93$ & $21.47 \pm 6.54$ & $22.33 \pm 8.20$ \\
\hline SSFEC (s) & $32.69 \pm 11.23$ & $32.29 \pm 10.12$ & $29.39 \pm 11.20$ & $28.13 \pm 10.26$ \\
\hline Sit-up (times $\min$ ) & $16.76 \pm 5.89$ & $16.79 \pm 7.39$ & $6.58 \pm 3.27$ & $5.77 \pm 2.75$ \\
\hline
\end{tabular}

Note: Pulse Pressure (PP), Diastolic Blood Pressure

Closing (SSFEC)

(DBP), Systolic Blood Pressure (SBP), Vital

4.1.3 Comparative Analysis on Body Physique Capacity/Weight Index (VCWI), Steps Experiment (SE), sit-and-reach (SR), Standing with a Single Foot and Eyes Indexes of Referential Group and Experimental Group before and after the Experiment

Table 5: Results of the Body Physique Indexes of Males of Referential Group and Experimental Group (M \pm SD)

\begin{tabular}{|c|c|c|c|}
\hline \multirow[b]{2}{*}{$\begin{array}{l}\text { Physiquew } \\
\text { Index }\end{array}$} & \multicolumn{3}{|c|}{ Males } \\
\hline & $\begin{array}{l}\text { Referential Group } \\
\text { after Experiment } \\
\qquad(\mathrm{n}=37)\end{array}$ & $\begin{array}{l}\text { Experimental Group } \\
\text { before Experiment } \\
(n=40)\end{array}$ & $\begin{array}{l}\text { Experimental Group } \\
\text { after Experiment } \\
(n=35)\end{array}$ \\
\hline Height $(\mathrm{cm})$ & $171.98 \pm 6.76$ & $170.98=6.06$ & $170.85 \pm 5.92$ \\
\hline Weight (kg) & $63.19 \pm 9.35$ & $60.24 \pm 9.05$ & $60.44 \pm 9.52$ \\
\hline $\mathrm{BMI}\left(\mathrm{kg} / \mathrm{m}^{2}\right)$ & $21.34 \pm 3.93$ & $20.57 \pm 3.63$ & $20.71 \pm 4.12$ \\
\hline Waist & $73.22 \pm 5.21$ & $73.22 \pm 5.21$ & $70.32 \pm 4.92$ \\
\hline Hip & $86.71 \pm 6.14$ & $86.76 \pm 6.14$ & $86.82 \pm 3.19$ \\
\hline WHR & $\begin{array}{c}0.84 \pm 0.23 \\
* *\end{array}$ & $\begin{array}{c}0.84 \pm 0.21 \\
* *\end{array}$ & $0.81 \pm 0.24$ \\
\hline $\mathrm{F} \%$ & $24.12 \pm 10.89$ & $24.12 \pm 10.89$ & $23.73 \pm 9.82$ \\
\hline
\end{tabular}

Note: F\% represents a sum of the upper-arm sebum thickness and scapular sebum thickness; * means $\mathrm{p} \leqslant$ 0.05 and a significant difference; $* *$ means $\mathrm{p} \leqslant 0.01$ and a highly significant difference.

After four months of the "Health Qigong•WuQinxi" exercise, the results of the body physique indexes of males of referential group and experimental group in table 5 showed there was a highly significant difference in the WHR within the groups and between the groups $(\mathrm{p} \leq 0.01)$; the waists within the groups and between the groups changed positively; and the differences between other data were insignificant. However, there was a significant difference in the WHR and waist of females 
within the groups and between the groups $(\mathrm{p} \leq 0.05)$; and

well.

the differences between other data were insignificant as

Table 6: Results of the Body Physique Indexes of Females of Referential Group and Experimental Group (M \pm SD)

\begin{tabular}{|c|c|c|c|}
\hline \multirow[b]{2}{*}{$\begin{array}{l}\text { Physique } \\
\text { Index }\end{array}$} & \multicolumn{3}{|c|}{ Females } \\
\hline & $\begin{array}{l}\text { Referential Group } \\
\text { after Experiment } \\
\qquad(\mathrm{n}=39)\end{array}$ & $\begin{array}{l}\text { Experimental Group } \\
\text { before Experiment } \\
(\mathrm{n}=40)\end{array}$ & $\begin{array}{l}\text { Experimental Group } \\
\text { after Experiment } \\
(\mathrm{n}=33)\end{array}$ \\
\hline Height (cm) & $159.16 \pm 3.58$ & $159.46 \pm 5.56$ & $159.32 \pm 5.50$ \\
\hline Weight (kg) & $53.36 \pm 7.82$ & $51.25 \pm 7.32$ & $51.08 \pm 6.98$ \\
\hline $\mathrm{BMI}\left(\mathrm{kg} / \mathrm{m}^{2}\right)$ & $21.06 \pm 3.29$ & $20.16 \pm 2.26$ & $20.19 \pm 3.21$ \\
\hline Waist & $65.33 \pm 2.31 *$ & $63.59 \pm 2.31$ & $62.08 \pm 2.19$ \\
\hline Hip & $90.42 \pm 5.38$ & $88.32 \pm 4.28$ & $88.21 \pm 4.52$ \\
\hline WHR & $0.72 \pm 0.19 *$ & $0.72 \pm 0.25 *$ & $0.70 \pm 0.21$ \\
\hline F\% & $34.98 \pm 10.86$ & $34.98 \pm 10.86$ & $34.74 \pm 10.29$ \\
\hline
\end{tabular}

Note: F\% represents a sum of the upper-arm sebum thickness and scapular sebum thickness; * means $\mathrm{p} \leqslant 0.05$ and a significant difference; $* *$ means $\mathrm{p} \leqslant 0.01$ and $\mathrm{a}$ highly significant difference.
4.1.4 Comparative Analysis on Physiological Indexes of Referential Group and Experimental Group before and after the Experiment

Table 7: Results of Physiological Indexes of Males of Referential Group and Experimental Group (M \pm SD)

\begin{tabular}{|c|c|c|c|}
\hline & & Males & \\
\hline $\begin{array}{c}\text { Physiological } \\
\text { Index }\end{array}$ & $\begin{array}{l}\text { Referential Group } \\
\text { after Experiment } \\
(n=37)\end{array}$ & $\begin{array}{l}\text { Experimental Group } \\
\text { before Experiment } \\
\qquad(n=40)\end{array}$ & $\begin{array}{l}\text { Experimental Group } \\
\text { after Experiment } \\
(n=35)\end{array}$ \\
\hline Pulse (times $/ \mathrm{min}$ ) & $81.39 \pm 7.96 *$ & $81.12 \pm 7.44 *$ & $78.56 \pm 7.25$ \\
\hline $\mathrm{PP}(\mathrm{mmHg})$ & $41.29 \pm 10.19$ & $42.12 \pm 11.59$ & $41.29 \pm 10.28$ \\
\hline $\mathrm{DBP}((\mathrm{mmHg})$ & $121.22 \pm 10.36 *$ & $121.12 \pm 10.29 *$ & $115.25 \pm 10.57$ \\
\hline $\mathrm{SBP}(\mathrm{mmHg})$ & $74.78 \pm 8.36$ & $76.89 \pm 8.12 *$ & $72.12 \pm 9.56$ \\
\hline VCWI & $51.75 \pm 11.29 * *$ & $51.26 \pm 10.29 * *$ & $56.79 \pm 11.33$ \\
\hline SE & $43.19 \pm 5.72 * *$ & $43.27 \pm 5.21 * *$ & $50.59 \pm 5.25$ \\
\hline $\mathrm{SR}(\mathrm{cm})$ & $7.89 \pm 8.29$ & $7.82 \pm 7.23$ & $9.52 \pm 8.17$ \\
\hline Grip (kg) & $38.28 \pm 8.93 *$ & $38.92 \pm 7.83 *$ & $42.33 \pm 8.26$ \\
\hline SSFEC (s) & $32.29 \pm 10.12$ & $31.29 \pm 10.12$ & $34.54 \pm 10.77$ \\
\hline Sit-up (times $/ \mathrm{min}$ ) & $16.79 \pm 7.39$ & $16.23 \pm 4.79$ & $16.55 \pm 6.89$ \\
\hline
\end{tabular}

Note: * means $\mathrm{p} \leqslant 0.05 \&$ a significant difference; *

means $\mathrm{p} \leqslant 0.01 \&$ a highly significant difference. 
Table 8: Results of Physiological Indexes of Females of Referential Group and Experimental Group (M \pm SD)

\begin{tabular}{|c|c|c|c|}
\hline \multirow[b]{2}{*}{$\begin{array}{l}\text { Physiological } \\
\text { Index }\end{array}$} & \multicolumn{3}{|c|}{ Females } \\
\hline & $\begin{array}{l}\text { Referential Group } \\
\text { after Experiment } \\
(\mathrm{n}=39)\end{array}$ & $\begin{array}{l}\text { Experimental Group } \\
\text { before Experiment } \\
\qquad(n=40)\end{array}$ & $\begin{array}{l}\text { Experimental Group } \\
\text { after Experiment } \\
(\mathrm{n}=33)\end{array}$ \\
\hline Pulse (times $\mathrm{min}$ ) & $83.17 \pm 9.43 *$ & $83.52 \pm 8.57 *$ & $79.96 \pm 8.19$ \\
\hline $\mathrm{PP}(\mathrm{mmHg})$ & $39.07 \pm 10.35$ & $40.02 \pm 10.25$ & $39.89 \pm 11.18$ \\
\hline $\mathrm{DBP}((\mathrm{mmHg})$ & $114.72 \pm 9.59$ & $114.22 \pm 9.17$ & $110.59 \pm 10.29$ \\
\hline $\mathrm{SBP}(\mathrm{mmHg})$ & $72.75 \pm 9.98 *$ & $72.15 \pm 7.89 *$ & $67.58 \pm 8.10$ \\
\hline VCWI & $42.73 \pm 10.38 * *$ & $40.33 \pm 10.18 * *$ & $48.37 \pm 10.92$ \\
\hline SE & $40.38 \pm 5.29 *$ & $40.18 \pm 4.27 *$ & $44.72 \pm 3.18$ \\
\hline $\mathrm{SR}(\mathrm{cm})$ & $13.22 \pm 5.88$ & $11.72 \pm 5.97 *$ & $16.39 \pm 6.24$ \\
\hline Grip (kg) & $22.33 \pm 8.20$ & $21.53 \pm 6.10$ & $23.17 \pm 5.59$ \\
\hline SSFEC (s) & $28.13 \pm 10.26$ & $27.17 \pm 9.27$ & $30.27 \pm 10.55$ \\
\hline Sit-up (times $/ \mathrm{min}$ ) & $5.77 \pm 2.75$ & $5.17 \pm 2.35$ & $5.28 \pm 2.67$ \\
\hline
\end{tabular}

Note: * means $\mathrm{p} \leqslant 0.05$ and a significant difference; $* *$ means $\mathrm{p} \leqslant 0.01$ and a highly significant difference.

After four months of the "Health Qigong•WuQinxi" exercise, the results of physiological indexes of males of referential group and experimental group is showed in table 7.

The "Health Qigong•WuQinxi" is imitating the manner, movement and mind of animals to stretch bones and muscles and unblock main and collateral channels, so as to recuperate organs and all circulation systems of human body. The exercise of "Health Qigong•WuQinxi" values the regulation of the breathing and aspiration, requiring an enhancement to the breath depth and cycle, and trying to reach a "fine, deep, long and balance" target as far as possible. Also, the "Health Qigong•WuQinxi" is an all-round exercise of human body, including bending forward, backward and sideward, twisting, etc. It exerts a good stretching role in the chest, waist, leg muscle and ligaments, and hence can enlarge the movement range of the spinal column, waist and legs. Also, "Health Qigong•WuQinxi" pays special attention to the movement of finger joints, so as to strengthen remote blood circulations. "Health Qigong•WuQinxi" imitates the movements and postures of the five animals, to stretch body and flex muscles and bones, to make it balanced and receive the minds of the five animals, which is a dynamic and static combination and hence enhances the balanced capacity of human body. The difference before and after the exercise of sit-ups was insignificant in the experimental group, suggesting the "Health Qigong•WuQinxi" exerts an insignificant impact on the abdominal muscle strength of human body, or needs a long time of practice.

\section{Conclusion}

The "Health Qigong•WuQinxi" is an excellent traditional Chinese culture, and properly combines the body movements, breathing and aspiration and psychological adjustment together, which is suitable for the students at universities to practice. Besides, in accordance with the results of the experimental research, there was a significant difference in the WHR, vital capacity/weight index and steps experiment index of the 
experimental participants before and after the four months of "Health Qigong•WuQinxi" exercises $(\mathrm{p} \leq 0.01)$; there was also a comparatively significant difference in their waist, pulse, systolic blood pressure and grip $(\mathrm{p} \leq 0.05)$; and there was a positive change in both the standing with a single foot and eyes closing and the sit-and-reach. All these suggest the Health Qigong can effectively improve the physical health of students at universities.

\section{Suggestions}

The "Health Qigong•WuQinxi" owns a rich cultural connotation and unique fitness values, and has been introduced into the classrooms of universities with a gradual step, and also has become a primary sport item of the physical rehabilitation course. Moreover, the "Health Qigong•WuQinxi" is an aerobic exercise with moderate and low intensities, which can try combining with other physical education courses with a high intensity, such as taekwondo and basketball, or can be used as the warming-up exercises or cooling-down exercises of these courses. Thus, a dynamic and static combination can be achieved in the physical educational courses. Finally, the physical educational teachers at universities can support and help their students to establish a "Health Qigong•WuQinxi" association, so as to bring the strengths of the students into play, communicate the health Qigong culture, and carry on and promote the excellent traditional culture.

\section{REFERENCES}

[1] Wang Ying, Lin Li. College Physical Education and Health Tutorial [M]. Shanghai: Tongji University Press, 2007: 81.

[2] Taoshixiubang. Metabolic Features of Mesenteric Fat and Visceral Fat Obesity [J]. The Lipid, 1994, 5:371-375.

[3] Tadashi Nakamura. Visceral Fat Diseases [J]. The Lipid, 1994, 5: 402-408.

[4] Qu Guangfu, Zhan Shengwei, et al. The Correlation between Lung Function and Body Mass Index, Waist, Waist-to-Hip Ratio in Adolescents [J]. 2008, 29 (8): 19-21.

[5] Wang Ruiyuan. Sports Physiology [M]. People's Sports Publishing House, 2002: 119.

[6] Research Group of Physical Health Standard of Students. Standard for the Physical Health of Students (on Trial) [M]. Beijing: People's Education Press, 2002: 84. 